\title{
HURST COEFFICIENT IN LONG TIME SERIES OF POPULATION SIZE: MODEL FOR TWO PLANT POPULATIONS WITH DIFFERENT REPRODUCTIVE STRATEGIES
}

\author{
ALFONSO GARMENDIA \\ Instituto Agroforestal Mediterráneo \\ Escuela Técnica Superior de Agronomia y Medio Natural \\ Universidad Politécnica de Valencia \\ Camino de Vera $s / n, 46022$ Valencia, Spain \\ algarsal@upvnet.upv.es \\ LUIS GARMENDIA \\ Department of Programming and Computing Systems \\ Complutense University of Madrid, Spain \\ lgarmend@fdi.ucm.es \\ ADELA SALVADOR \\ Technical University of Madrid \\ E.T.S.I. Caminos, Spain \\ ma09@caminos.upm.es
}

\begin{abstract}
Can the fractal dimension of fluctuations in population size be used to estimate extinction risk? The problem with estimating this fractal dimension is that the lengths of the time series are usually too short for conclusive results. This study answered this question with long time series data obtained from an iterative competition model. This model produces competitive extinction at different perturbation intensities for two different germination strategies: germination of all
\end{abstract}


seeds vs. dormancy in half the seeds. This provided long time series of 900 years and different extinction risks. The results support the hypothesis for the effectiveness of the Hurst coefficient for estimating extinction risk.

Keymords: Homocarpy; Heterocarpy; Fractal Dimension; Extinction; Time Series; Hurst Coefficient.

\section{INTRODUCTION}

This article is the continuation of two previous articles. ${ }^{1,2}$ In "Fractal Dimension of Birds Population Sizes Time Series," 1 a fractal was defined as an object whose topological dimension does not coincide with the Hausdorff dimension. The time series dimension was studied, and different methods to obtain the Hurst coefficient $(H)$ were offered. $H$ was used in time series of Passeriforme populations to analyze its usefulness as an indicator of extinction risk. The main problem in the above study was that the 20 years time series used are too short for a good $H$ estimate, and therefore it was found that longer time series were needed to study $H$ 's as an extinction indicator.

"The importance of the intensity and frequency of perturbations on the germination delay" ${ }^{2}$ studied a model in which two plant populations with different reproductive strategies competed for a territory. The model evaluated perennial plants, considering perturbations as the only cause of death. These perturbations were defined by two parameters: occurrence probability $(O P)$ and perturbation intensity $(P I)$.

$H$ has been used to estimate fractal dimension ${ }^{3-7}$ to study a variety of problems in ecology ${ }^{8-12}$ and biology ${ }^{13-18}$ High $H$ values can be interpreted as extinction risk. ${ }^{7}{ }^{18}$ Hastings and Sugihara ${ }^{19}$ suggested that increased range of fluctuations with the time interval might mean an increased likelihood of extinction. The main problem of estimating $H$ for natural populations is that time series are always very short, and therefore sometimes it is difficult even to demonstrate their fractal structure. Iterative population models provide an oportunity to check this hypothesis without this problem.

In this paper, the time series obtained in Ref. 2 were used to analyze $H$ to test its effectiveness for extinction risk estimates.

This work aims to verify whether $H$ is a good indicator of species extinction based on:

(1) the use of long time series (900 years), sufficiently long for reliable measurement of $H$; and
(2) different environments, defined by different perturbation frequencies and intensities that produce differences in extinction risk.

The study objectives therefore are, firstly, to analyze variations in $H$ for the two germination strategies under different perturbation frequencies and intensities. Secondly, to compare time series prior to extinction and series without extinction, and to check whether differences in $H$ enable prediction of species extinction.

\section{MATERIAL AND METHODS}

\subsection{Brief Description of the Iterative Model}

See Ref. 2 for a full explanation of the model used to obtain the time series. This iterative model was used to compare the effect of perturbation on two different reproductive strategies, homocarpic plants (whose seeds germinate the first year) and heterocarpic plants (that form a seed bank, from which only half of them germinate each year) competing for a territory. The model is used to examine the adaptability of these strategies to different perturbations. Seeds that germinate must find and occupy an empty place in a particular cell in a grid and thus become an adult plant, otherwise they die.

Perturbations were the only cause of death considered for adult plants, using random variables with a chosen probability, $O P$ that indicates perturbation occurrence probability, and $P I$ that is defined as the likelihood of death for each plant when the perturbation occurs.

In Ref. 2, it was concluded that heterocarpy is a competitive strategy in perturbed environments, as heterocarpic plants were dominant at high $P I$, and that the extinction of homocarpic plants was more dependent on $P I$ than $O P$. In the present study, therefore, $H$ was analyzed at different $P I$ values, keeping $O P$ at 0,5 .

The study area consisted of 100 cells distributed in a $10 \times 10$ toroidal grid, i.e. avoiding the presence of borders, and the independent variables were fixed 
for all the time series: the number of seeds produced by each adult plant was assumed to be three per year, randomly dispersed between the plant's own cell and the eight neighbouring cells. Five was the maximum number of adult plants in each cell.

\subsection{Programs}

Two computer programs were used for this paper, but both has already been used before: To obtain the $H$ values, the program called "Hurst Coefficient" (HC) from Ref. 1 was used, and to obtain the long time series (1000 years) at different perturbation intensities (and therefore, different extinction risks), the program "Time series" (TS) was used. ${ }^{2}$

For each $P I$ and each $O P$ value from $0.1-0.9$, in steps of 0.1 , the TS program was run iteratively to obtain $10 \times 1000$ years time series. The first 100 years of each time series was eliminated so that the series only represent the phase in which populations have achieved a certain independence from initial conditions. This gave time series of 900 years if the species did not become extinct or less (up to the year of extinction) for the cases where the species became extinct.

These time series were entered in the $\mathrm{HC}$ program to calculate $H$. This program calculates $H$ from several methods, but for clarity's sake, only one was chosen: the range increment method. Different methods can produce different results, but there is a linear relationship between the results of all of them ${ }^{1,12}$ and consequently no variation of the conclusions would emerge from which method is used. Nevertheless, results from second order and local methods were also compared but they are not. included because trends were very similar.

The range increment method was chosen because it is more closely related to the theoretical basis for using $H$ to estimate extinction risk. ${ }^{12}$ The range is the difference between the maximum and minimum values in the time series in a given time interval.

$H$ variability was calculated by running the program ten times for each $O P$ and $P I$ value and obtaining the average and standard deviation from the ten $H$ estimates. To observe the changes caused by $P I$ (the factor which increases the extinction risk) in $H$, measurements were taken from $P I$ of $0.1-0.9$ in steps of 0.1 .

The HC program ${ }^{1}$ is implemented in Pascal and can be downloaded free of charge from: http://personales.upv.es/ algarsal/hurst.zip
The TS program ${ }^{2}$ that simulated competition for space between plants with homocarpic and heterocarpic reproductive strategies was implemented in $\mathrm{C}++$ and can be seen at: http://personales.upv .es/ algarsal/plantas.zip

\subsection{Hurst Coefficient Range Increment Method}

The range increment method obtains $H$ by the expression $R(\Delta t)=c \Delta t^{H}$, where $\Delta t$ is a given time step, and $R(\Delta t)$ denotes average values in the process range $\{y(t)\}$, for all time steps of duration $\Delta t$

To test the hypothesis that the scaling is applicable, tests of linearity has been made for several time increments (an example can be seen at Fig. 1). For comparison between different perturbation intensities and plant strategies, the time increment $\Delta t$ was set at 10 and 100 .

$H$ measured by the range increment method provides a measure of increase in the range of fluctuations in a time series as the time interval is increased, which could be an indicator of extinction risk. ${ }^{12}$ Hastings and Sugihara ${ }^{19}$ suggested that as the range grows, fluctuations increase and so does the extinction risk. They also observed that $H$ calculated by this method in short time series could be higher than the real value.

As species sometimes become extinct in a period of 200 years (i.e. within the time series used) $H$ could not be calculated by the range increment method using $\Delta t=100$, and therefore the results are analysed using the range increment method with $\Delta t=10$.

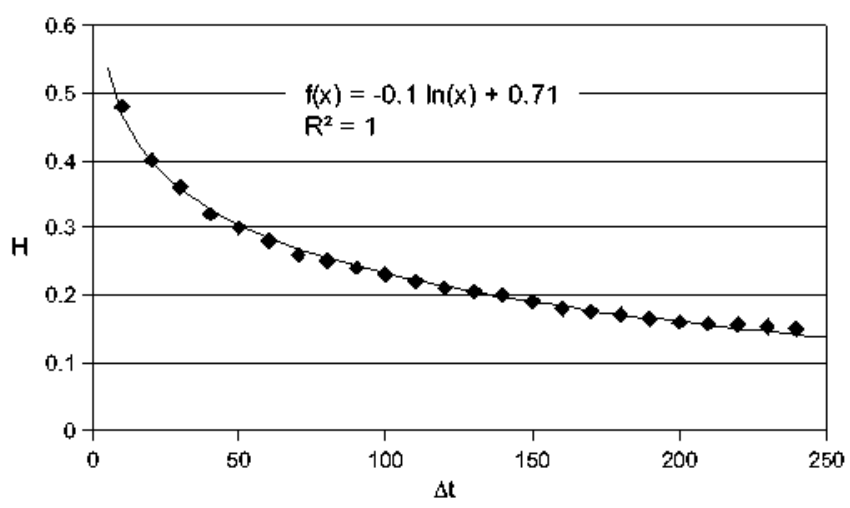

Fig. 1 Linearity test for the Hurst coefficient $(H)$ measured for the heterocarpic population with $P I=0.8, O P=0.8$ ( $P I$, perturbation intensity; $O P$, occurrence probability for perturbation). 
ANOVA was used to analyze $H$ variability in the ten time series for each given value of $O P$ and $P I$, to evaluate the differences, and measurements were compared using Student's t-test.

\section{RESULTS}

\subsection{Range Fluctuations Analysis}

In Ref. 2 it was found that range values were affected by $P I$ with little influence by the perturbation frequency.

Fluctuation range behaviour in homocarpic and heterocarpic plants in 1000 years time series with $O P=0.5$ and for different $P I$ values is shown in Fig. 2.

For $P I=0.2$ the fluctuation range was around 100 plants; for homocarpic plants $P I$ was

$\mathrm{PI}=0.2$ Homocarpic

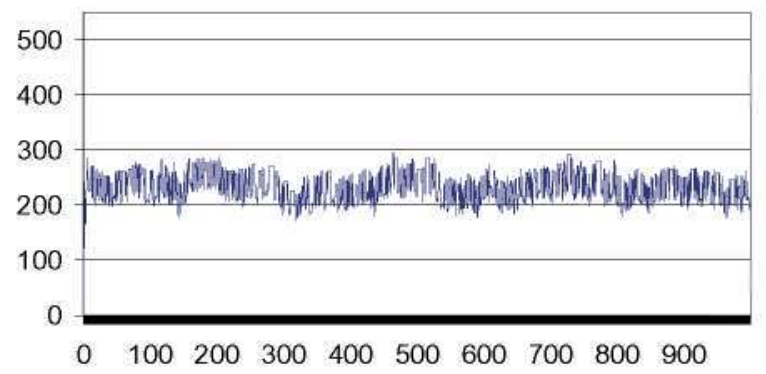

$\mathrm{PI}=0.5$ Homocarpic

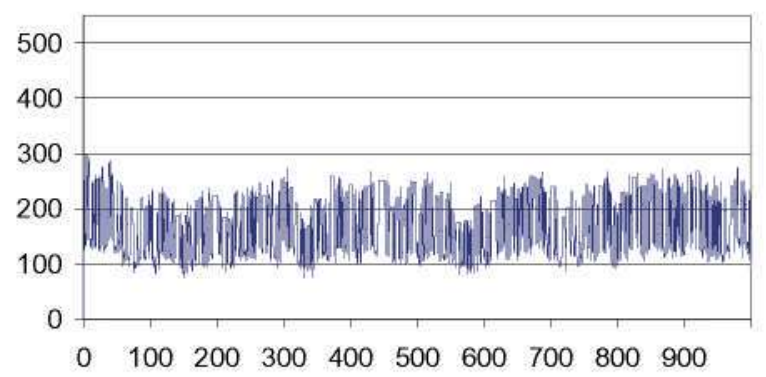

$\mathrm{PI}=0.8$ Homocarpic

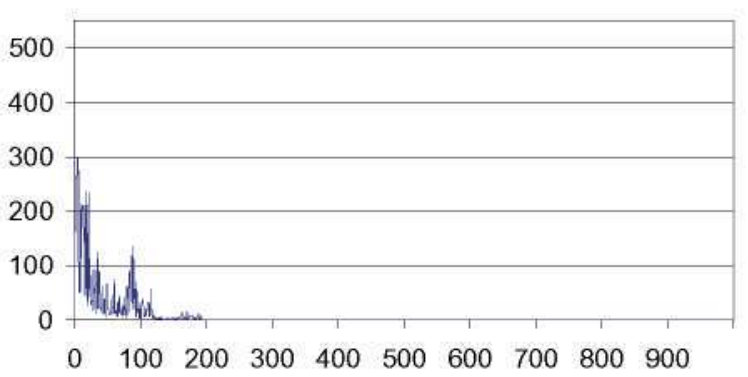

approximately 200-300 plants, and heterocarpic 150-250 plants. For $P I=0.5$ the fluctuation range was around 200 plants; for homocarpic plants $P I$ was 100-250 plants and for heterocarpic 100-300 plants. For $P I=0.8$ homocarpic plants became extinct within 200 years, and due to lack of competition, the number of heterocarpic plants increased, with wide fluctuations of $100-500$ plants. The seed bank strategy favoured plants in environments with very intense perturbations.

\subsection{Hurst Coefficients Measured by the Range Increment Method for Different $O P$ and $P I$ Values}

Table 1 shows the averages and standard deviations for $H$ in ten time series of homocarpic and
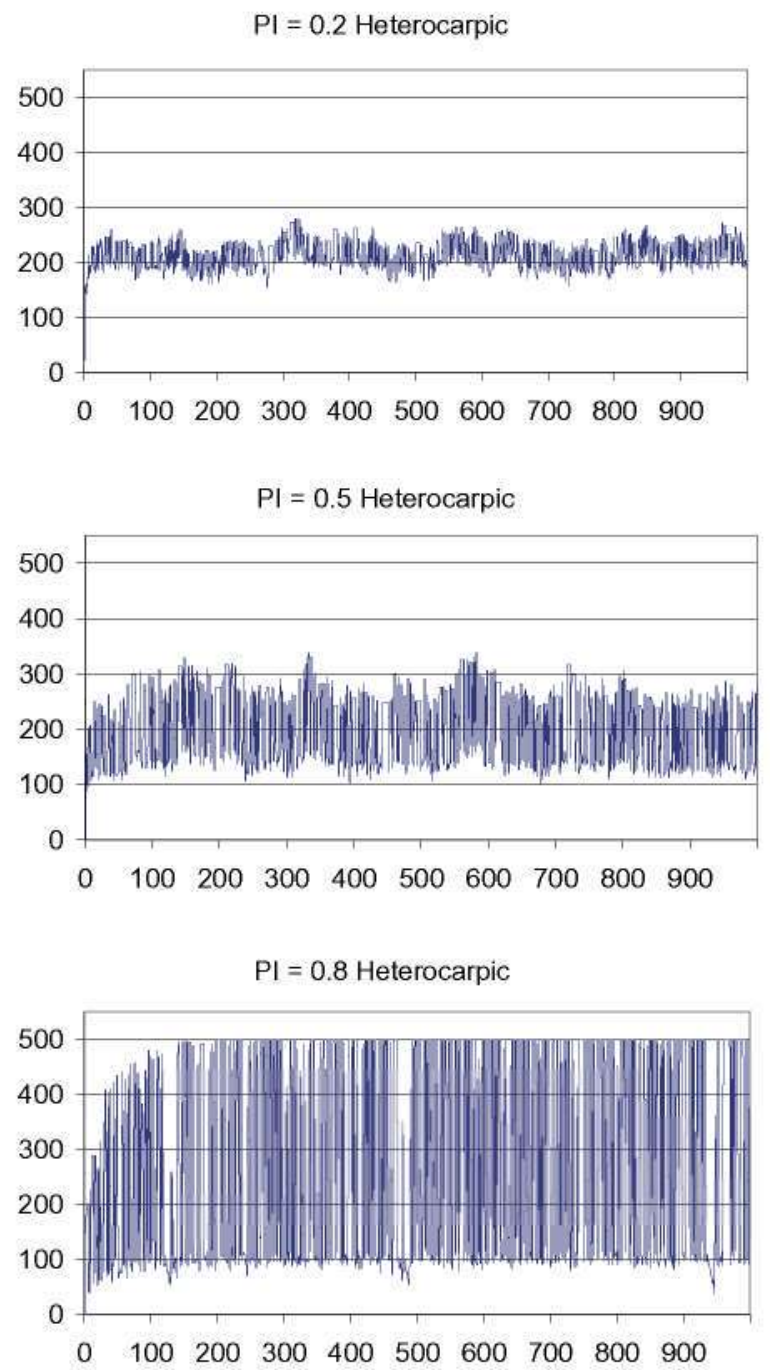

Fig. 2 A 1000 years temporal series for different $P I$ with $O P=0.5$, and different reproductive strategies, homocarpic and heterocarpic. Each pair of graphs represents one particular case. Increasing fluctuations in amplitude are evident with higher $P I$ values ( $P I$, perturbation intensity; $O P$, occurrence probability for perturbation). 
Table 1 The Range Increment Method Provided the Hurst Coefficient $(H)$ for the Equation: $R(\Delta t)=c \Delta t^{H}$, where $\Delta t$ is the time-step, $R(\Delta t)$ denotes the average range value of the process $\{y(t)\}$, at all time intervals of duration $\Delta t$. Range is the difference between the maximum and the minimum values of $y(t)$. This table shows the average $H$ in ten different time series for homocarpic and heterocarpic strategies with different $P I$ and $O P$ values. The measures were made by the range method with $\Delta t=100$ years and $\Delta t=10$ years. Error was measured as the standard deviation for ten different time series. Different letters on $H$ measures represent significant mean differences $(P<0.05)(P I$, perturbation intensity; $O P$, occurrence probability for perturbation).

\begin{tabular}{lrll}
\hline \multicolumn{5}{c}{$O P=0.2$} & \multicolumn{1}{c}{$O P=0.5$} & \multicolumn{1}{c}{$O P=0.8$} \\
\hline Homocarpic plants $(\Delta t=100)$ & & \\
$P I=0.2$ & $0.27 \pm 0.01 \mathrm{e}$ & $0.20 \pm 0.00 \mathrm{~b}$ & $0.27 \pm 0.01 \mathrm{e}$ \\
$P I=0.5$ & $0.23 \pm 0.01 \mathrm{c}$ & $0.15 \pm 0.01 \mathrm{a}$ & $0.20 \pm 0.01 \mathrm{~b}$ \\
$P I=0.8$ & $0.25 \pm 0.01 \mathrm{~d}$ & Extinct & Extinct \\
Heterocarpic plants $(\Delta t=100)$ & & \\
$P I=0.2$ & $0.29 \pm 0.01 \mathrm{~g}$ & $0.21 \pm 0.00 \mathrm{~d}$ & $0.28 \pm 0.01 \mathrm{~g}$ \\
$P I=0.5$ & $0.23 \pm 0.01 \mathrm{f}$ & $0.15 \pm 0.01 \mathrm{~b}$ & $0.19 \pm 0.01 \mathrm{c}$ \\
$P I=0.8$ & $0.23 \pm 0.01 \mathrm{f}$ & $0.08 \pm 0.01 \mathrm{a}$ & $0.22 \pm 0.01 \mathrm{e}$ \\
Homocarpic plants $(\Delta t=10)$ & & $0.46 \pm 0.02 \mathrm{c}$ \\
$P I=0.2$ & $0.52 \pm 0.02 \mathrm{e}$ & $0.37 \pm 0.03 \mathrm{ab}$ & $0.45 \pm 0.02 \mathrm{c}$ \\
$P I=0.5$ & $0.49 \pm 0.02 \mathrm{~d}$ & $0.33 \pm 0.04 \mathrm{a}$ & $0.52 \pm 0.02 \mathrm{Extinct}$ \\
$P I=0.8$ & $0.51 \pm 0.02 \mathrm{e}$ & $0.40 \pm 0.03 \mathrm{Extinct} \mathrm{b}$ & \\
Heterocarpic plants $(\Delta t=10)$ & & $0.46 \pm 0.02 \mathrm{c}$ \\
$P I=0.2$ & $0.52 \pm 0.01 \mathrm{e}$ & $0.37 \pm 0.03 \mathrm{~b}$ & $0.46 \pm 0.02 \mathrm{c}$ \\
$P I=0.5$ & $0.49 \pm 0.02 \mathrm{~d}$ & $0.33 \pm 0.04 \mathrm{ab}$ & $0.48 \pm 0.02 \mathrm{~cd}$ \\
$P I=0.8$ & $0.48 \pm 0.02 \mathrm{~cd}$ & $0.30 \pm 0.04 \mathrm{a}$ & \\
\hline
\end{tabular}

heterocarpic plants for 900 years (1000 years minus the first 100 years) of competition for each different $P I$ and $O P$. For example, for $P I=0.8$ and $O P=0.2$ from the range increment method with $\Delta t=10$, in homocarpic plants, the mean value of $H=0.51$ was calculated from ten time series of 900 years each. The standard deviation was 0.01 .

$H$ had uniform values for each of the ten time series used in each case, as the typical deviations were all very low $(<0.05)$. These typical deviations were lower (and the differences clearer) for $\Delta t=100$ than for $\Delta t=10$, although the general behaviour was very similar.

For $P I=0.8$ and $O P=0.5$ homocarpic plants became extinct in about 200 years and $H=0.4$ for the time series before extinction. For $O P=0.8$ and $P I=0.8, H=0.52$, and therefore, $H$ increased with increased $P I$. At the same time, $H$ for heterocarpic populations was significantly lower $(P<0.05)$ when the homocarpic population became extinct.

The highest $H$ values $(\Delta t=10$ ) for homocarpic plants were obtained from $O P=0.5, P I=0.8(H=$ $0.40)$ and $O P=0.8, P I=0.8(H=0.52)$, which suggests that $H$ is a good indicator of extinction risk. A similar observation applied to $\Delta t=100$.

\subsection{Study of $H$ for the $P I$ Variable}

For the results in Table 1, the average values for ten time series of $H$ obtained by the range increment method for $\Delta t=10$ and $\Delta t=100$, for different values of $P I$ and $O P=0.5$, are found in Fig. 3 .

When $P I$ increased, $H$ decreased, fitting a straight line in heterocarpic plants. There was a very different shape for homocarpic plants, which decreased initially to a minimum of $P I=0.5$ and then increased. $H$ increased when homocarpic plants showed an extinction risk.

\section{CONCLUSIONS}

The Hurst coefficient is a good indicator of extinction risk for the long 900 years time series used in this study. There was a strong relation between the $H$ and $P I$ as the fractal dimension of the time series and fluctuations decreased (lower $H$ ) with $P I$ in heterocarpic plants, which are the most resilient, bestadapted to perturbation species. In homocarpic plants, which are more sensitive to perturbations, the coefficient and fractal dimension reached high values for high $P I$ on situations when there was a high extinction risk. 


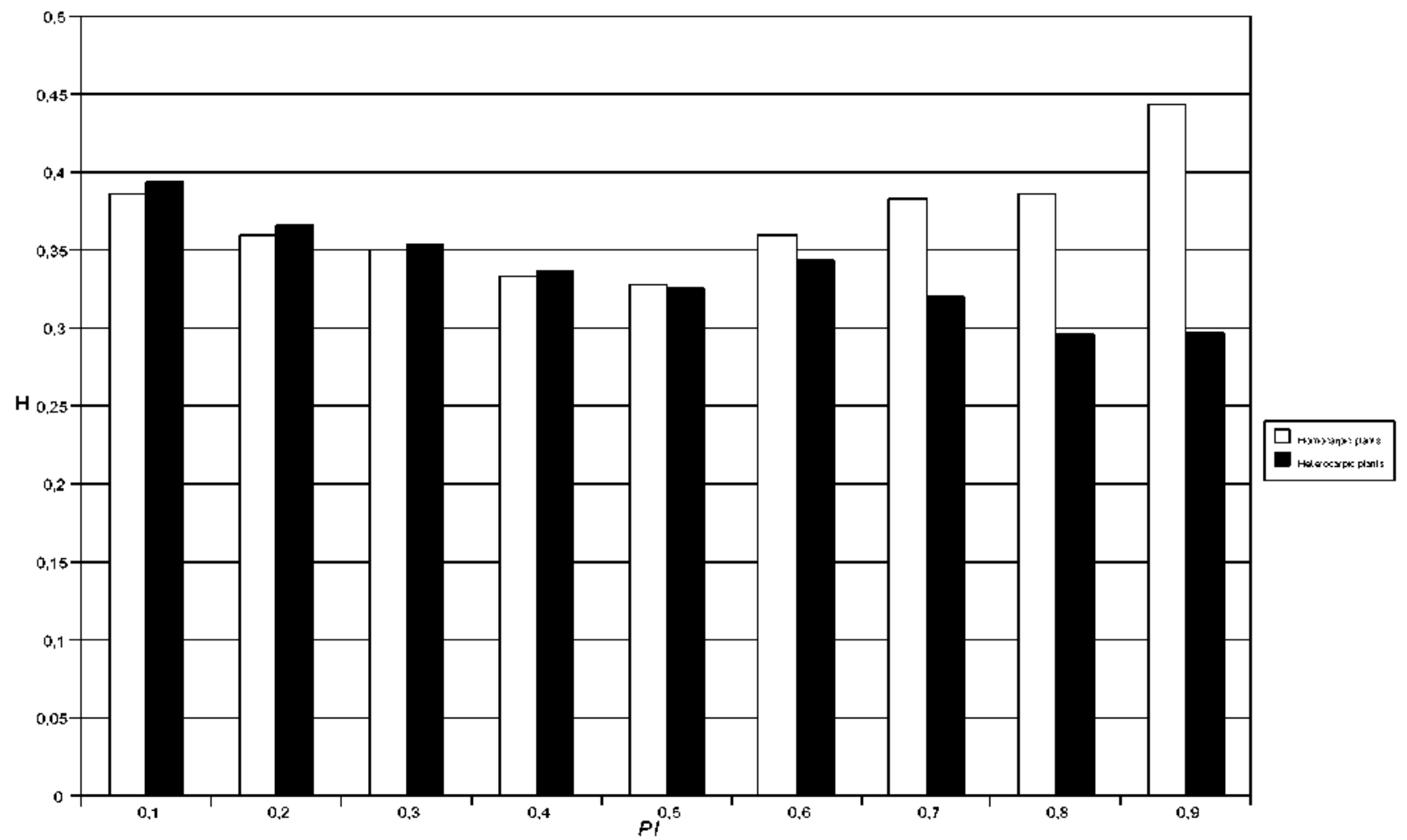

(a)

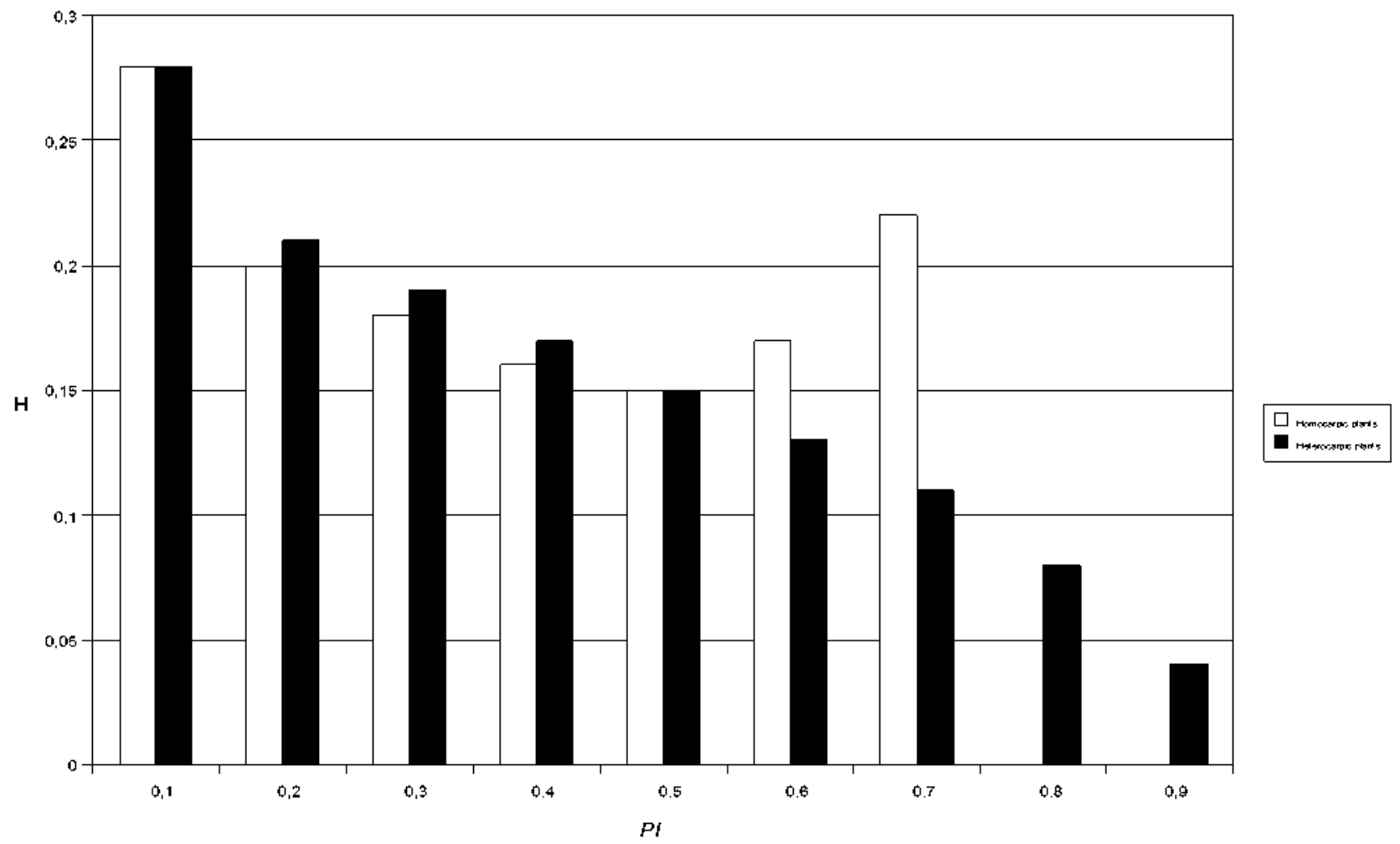

(b)

Fig. 3 The mean Hurst coefficient $(H)$ values for homocarpic and heterocarpic plants during 1000 years of competition with different $P I . O P=0.5$ for all cases. The first 100 years of the temporal series were eliminated to include only the "stable" dynamic. (a) Measured with $\Delta t=10$ years. (b) Measured with $\Delta t=100$ years, $H$ for $P I=0.8$ and $P I=0.9$ could not be measured, because extinction occurred in about 200 years ( $P I$, perturbation intensity; $O P$, occurrence probability for perturbation). 
In general, $H$ increased only when the extinction risk increased. No such increase occurred when there were no extinctions, even when perturbation conditions were intermediate or even intense. The lowest $H$ values occurred in heterocarpic plants when they were favoured by perturbations which eliminated competition (despite the fact that in such cases $P I$ was very high), and in homocarpic plants when there were moderate perturbations with no extinction risk.

\section{REFERENCES}

1. A. Garmendia and A. Salvador, Fractal dimension of birds population sizes time series, Math. Biosci. $206(2007) 155-171$.

2. A. Garmendia and A. Salvador, The importance of the intensity and frequency of perturbations on the germination delay, Math. Biosci. 211 (2008) 153165 .

3. B. B. Mandelbrot, Fractals: Form, Chance and Dimension (Freeman, San Francisco, 1977).

4. B. B. Mandelbrot, The Fractal Geometry of Nature, 2nd. edn. (W. H. Freeman \& Co., San Francisco, $1982)$.

5. A. Garmendia and A. Salvador, Fractal: Punto fijo de aplicaciones contractivas, in Proceeding of VI JAEM, (Badajoz 1993), pp. 37-47.

6. M. F. Barnsley, Fractals Everywhere (Academic Press Inc. Boston, 1988).

7. K. J. Falconer, The Geometry of Fractal Sets (C.U.P., Cambridge, 1988).

8. R. H. Bradbury, R. E. Reitschelt and D. G. Green, Fractals in ecology: Methods and interpretation, Marine Ecol. Prog. Ser, 14 (1984) 295-296.
9. C. D. Cutler, A review of the theory and estimation of fractal dimension, in Nonlnear Time Series and Chaos, ed. H. L. Tong (World Scientific, Singapore, 1993), Vol. I, Dimension Estimation and Models.

10. J. M. García-Ruíz and F. Otálora, El uso de la geometría fractal en las ciencias naturales, Epsilon 28 (1994) $109-126$.

11. D. R. Morse, J. H. Lawton, M. M. Dodson and M. H. Willianson, Fractal dimension of vegetation and the distribution on anthropod body lengths, Nature $\mathbf{3 1 4}$ (1985) 731-733.

12. G. Sugihara and R. M. May, Applications of fractal in ecology, Trends Ecol. Evol. 5(3) (1990) 79-86.

13. J. M. Pacheco, Fractales y oceanografía, Epsilon 28 (1994) 99-108.

14. T. Gisiger, Scale invariante in biology: Coincidence or footprint of a universal mechanism? Biol. Rev. $\mathbf{7 6}$ (2001) 161-209.

15. R. E. Plotnick and J. J. Sepkoski, A multiplicative multifractal model for originations and extinctions, Paleobiology 27 (2001) 126-139.

16. J. Huisman and F. J. Weissing, Fundamental unpredictability in multispecies competition, Am. Nat. 157 (2001) 488-494.

17. J. J. Lennon, W. E. Kunin and S. Hartley, Fractal species distributions do not produce power-law species-area relationships, Oikos $97(2002) 378-386$.

18. L. Borda-de-água, S. P. Hubbell and M. Mcallister, Species-area curves, diversity indices and species abundance distributions: A multifractal analysis, Am. Nat. 159 (2002) 138-155.

19. H. M. Hastings and G. Sugihara, Fractals: A User's Guide for the Natural Sciences (Oxford University Press, Oxford, 1993). 